\title{
Designs in Grassmannian Spaces and Lattices
}

\author{
CHRISTINE BACHOC \\ bachoc@math.u-bordeaux.fr \\ RENAUD COULANGEON \\ coulange@math.u-bordeaux.fr \\ Laboratoire A2X, Université Bordeaux I, 351, cours de la Libération, 33405 Talence, France
}

GABRIELE NEBE

nebe@mathematik.uni-ulm.de

Abteilung Reine Mathematik, Universität Ulm, 89069 Ulm, Germany

Received May 2, 2001; Revised February 19, 2002

\begin{abstract}
We introduce the notion of a $t$-design on the Grassmann manifold $\mathcal{G}_{m, n}$ of the $m$-subspaces of the Euclidean space $\mathbb{R}^{n}$. It generalizes the notion of antipodal spherical design which was introduced by P. Delsarte, J.-M. Goethals and J.-J. Seidel. We characterize the finite subgroups of the orthogonal group which have the property that all its orbits are $t$-designs. Generalizing a result due to B. Venkov, we prove that, if the minimal $m$-sections of a lattice $L$ form a 4-design, then $L$ is a local maximum for the Rankin function $\gamma_{n, m}$.
\end{abstract}

Keywords: lattice, Grassmann manifold, orthogonal group, zonal function

\section{Introduction}

The notion of strongly perfect lattice is due to Boris Venkov. It is a subclass of the extreme lattices, i.e. of the lattices on which the Hermite function is a local maximum, distinguished by a combinatorial property of its minimal vectors. Namely, its minimal vectors form a set which is a 4-spherical design in the sense of [4].

In this paper, we introduce an analogous notion of $t$-design on the Grassmann manifold $\mathcal{G}_{m, n}$ of the $m$-subspaces of the Euclidean space $\mathbb{R}^{n}$. To this aim we use the decomposition of the $O(n)$-module $L^{2}\left(\mathcal{G}_{m, n}\right)$ of square integrable functions on $\mathcal{G}_{m, n}$ into a sum of irreducibles and the calculation of unique elements in each of the irreducible subspaces as zonal functions which is performed in [8] (see Section 3). The next section gives various criteria for $t$ designs on $\mathcal{G}_{m, n}$; in particular we characterize the finite subgroups of $O(n)$ whose orbits on the grassmannians are always $t$-designs. If the minimal $m$-sections of a lattice $L$ form a 4-design, we call the lattice $L$ strongly m-perfect. Using the description of $m$-extreme lattices repeated in Section 5, we show that the strongly $m$-perfect lattices are $m$-extreme. The final section gives lower bounds for the minima of strongly $m$-perfect lattices.

\section{The Grassmann manifold}

The Grassmann manifold $\mathcal{G}_{m, n}$ is the manifold of $m$-dimensional subspaces of the Euclidean space $\mathbb{R}^{n}$. It is a homogeneous space for the action of the orthogonal group $O(n):=O\left(\mathbb{R}^{n}\right)$. The stabilizer of a given $m$-subspace $p$ is isomorphic to $O(m) \times O(n-m)$ (since an 
orthogonal transformation which preserves $p$ also preserves $p^{\perp}$ ). The manifold $\mathcal{G}_{m, n}$ is compact, and therefore endowed with its Haar measure $\mu$. The space of square-integrable real valued-functions $L^{2}\left(\mathcal{G}_{m, n}\right)$ is endowed with the usual scalar product

$$
\left\langle f_{1}, f_{2}\right\rangle=\int_{\mathcal{G}_{m, n}} f_{1}(x) f_{2}(x) d \mu(x)
$$

In order to parametrise the orbits of pairs of grassmannians under $O(n)$, we have to introduce principal angles between subspaces. We collect some rather well-known facts about this notion (see [2], or [6] p. 584). Let $m \leq q \leq n / 2$ and $\left(p, p^{\prime}\right) \in \mathcal{G}_{m, n} \times \mathcal{G}_{q, n}$. Denote by $\operatorname{pr}_{p}$ (resp. $\operatorname{pr}_{p^{\prime}}$ ) the orthogonal projection on $p$ (resp. $p^{\prime}$ ). As $v$ varies over $p^{\prime}$, the function $v \mapsto \frac{\left\|\operatorname{pr}_{p}(v)\right\|}{\|v\|}$ admits $m$ critical values $0 \leq t_{m}=\cos \theta_{m} \leq \cdots \leq t_{1}=\cos \theta_{1} \leq 1$. Moreover, one can construct orthonormal bases $\left\{u_{i}\right\}$ and $\left\{v_{i}\right\}$ of $p$ and $p^{\prime}$ such that $u_{i} \cdot v_{i}=t_{i}$ for $1 \leq i \leq m$, and $u_{i} \cdot v_{j}=0$ if $i \neq j$. Completing $\left\{u_{i}\right\}$ to an orthonormal basis $B$ of $\mathbb{R}^{n}$, and writing down the $n \times q$ generating matrix $M$ of $p^{\prime}$ in this basis, we get:

$$
M=\left(\begin{array}{ccccccc}
\cos \theta_{1} & 0 & \ldots & 0 & * & \ldots & * \\
0 & \cos \theta_{2} & \ldots & 0 & * & \ldots & * \\
\vdots & \vdots & & \vdots & \vdots & & \vdots \\
0 & 0 & \ldots & \cos \theta_{m} & * & \ldots & * \\
\sin \theta_{1} & 0 & \ldots & 0 & * & \ldots & * \\
0 & \sin \theta_{2} & \ldots & 0 & * & \ldots & * \\
\vdots & \vdots & & \vdots & \vdots & & \vdots \\
0 & 0 & \ldots & \sin \theta_{m} & * & \ldots & * \\
0 & 0 & \ldots & 0 & * & \ldots & * \\
\vdots & \vdots & & \vdots & \vdots & & \vdots \\
0 & 0 & \ldots & 0 & * & \ldots & *
\end{array}\right)
$$

In particular, the $m$-tuple $\left(\cos \theta_{1}, \ldots, \cos \theta_{m}\right)$ characterizes the $O(n)$-orbit of the pair $\left(p, p^{\prime}\right)$.

The $y_{i}:=\cos ^{2} \theta_{i}$ may be calculated as the first $m$ eigenvalues, in decreasing order, of

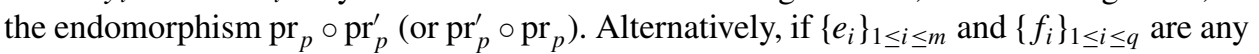
bases of $p$ and $p^{\prime}$, the $y_{i}$ are just the eigenvalues of the $m \times m$ matrix:

$$
\left(e_{i} \cdot e_{j}\right)^{-1}\left(e_{i} \cdot f_{j}\right)\left(f_{i} \cdot f_{j}\right)^{-1}\left(f_{i} \cdot e_{j}\right)
$$

\section{Decomposition of $L^{2}\left(\mathcal{G}_{m, n}\right)$ and intertwining functions}

In this section we decompose the $O(n)$-module $L^{2}\left(\mathcal{G}_{m, n}\right)$ into irreducible submodules. Replacing any subspace $p \in \mathcal{G}_{m, n}$ by its orthogonal complement, we may assume that $m \leq \frac{n}{2}$. We shall make use of several classical results of representation theory, for which 
we refer to the book of Goodman and Wallach [7] and Fulton and Harris [5]. Following [7], $F_{n}^{\mu}$ denotes the irreducible module of $\operatorname{GL}(n, \mathbb{R})$ of highest weight $\sum_{i=1}^{n} \mu_{i} L_{i}$, where $\mu$ is a partition $\mu=\mu_{1} \geq \mu_{2} \geq \ldots \mu_{n} \geq 0$ in at most $n$-parts (cf. [5, Proposition 15.15]).

Definition 3.1 The degree of a partition $\mu$ is $\operatorname{deg}(\mu):=\sum_{i} \mu_{i}$, and the depth of $\mu$ is $\operatorname{depth}(\mu):=\max \left\{i \mid \mu_{i} \neq 0\right\}$.

If $\operatorname{depth}(\mu) \leq \frac{n}{2}$, then the restriction of $F_{n}^{\mu}$ to the orthogonal group $O(n)$ contains a unique irreducible submodule $V_{n}^{\mu}$, which does not occur in the spaces $F_{n}^{\kappa}$, with $\operatorname{deg}(\kappa)<\operatorname{deg}(\mu)$. The representation $V_{n}^{\mu}$ is associated to the partition $\mu$ as described in [5, Section 19.5].

From [7, Section 12.3.2] (pp. 544-547) (where they show the analogous assertion for $S O(n))$ one gets the following theorem:

Theorem 3.2 Let $m \leq \frac{n}{2}$. Then the $O(n)$-space $L^{2}\left(\mathcal{G}_{m, n}\right)$ is isomorphic to

$$
L^{2}\left(\mathcal{G}_{m, n}\right) \simeq \bigoplus V_{n}^{\mu}
$$

where the sum is over the $\mu$ of depth at most equal to $m$, with all the $\mu_{i} \equiv 0 \bmod 2$.

Definition 3.3 Denote the unique submodule of $L^{2}\left(\mathcal{G}_{m, n}\right)$ isomorphic to $V_{n}^{\mu}$ by $H_{m, n}^{\mu}$.

Let $p_{0}$ be the $m$-subspace generated by the first $m$ elements of the canonical basis of $\mathbb{R}^{n}$. Then the stabilizer of $p_{0}$ in $O(n)$ is $\operatorname{Stab}\left(p_{0}\right) \cong O(m) \times O(n-m)$ and $L^{2}\left(\mathcal{G}_{m, n}\right)$ is the induced module from the trivial $O(m) \times O(n-m)$-module. Hence by Frobenius reciprocity $[7,12.1 .8]$ each of the irreducible $O(n)$-subspaces $H_{m, n}^{\mu}$ contains a (up to scalar multiples) unique zonal function $P_{\mu}$, i.e. a function that is invariant under $O(m) \times O(n-m)$.

In the following we give a more precise description of $H_{m, n}^{\mu}$ and explain a strategy used in [8] to calculate $P_{\mu}$.

We fix the following notations which we will keep for the rest of the paper: for all $m$, $n \in \mathbb{N}$, let $M_{n, m}$ denote the vector space of $n \times m$ matrices with real coefficients, and $S M_{m}$ the vector space of the real symmetric $m \times m$ matrices.

We consider the spaces of homogeneous polynomials of degree $k$ in the coefficients of these matrices, denoted by $\operatorname{Hom}_{k}\left(M_{n, m}\right)$ and $\operatorname{Hom}_{k}\left(S M_{m}\right)$. The group GL $(m, \mathbb{R})$ acts on $\operatorname{Hom}_{k}\left(S M_{m}\right)$ by $(g \cdot f)(S)=f\left(g^{t} S g\right)$, and the product $\operatorname{GL}(n, \mathbb{R}) \times \operatorname{GL}(m, \mathbb{R})$ acts on $\operatorname{Hom}_{k}\left(M_{n, m}\right)$ by $((g, h) \cdot f)(M)=f\left(g^{t} M h\right)$.

The decomposition of the $\operatorname{GL}(n, \mathbb{R})$-module $\operatorname{Hom}_{k}\left(S M_{n}\right)$ is given by ([7, Theorem 5.2.9]):

$$
\operatorname{Hom}_{k}\left(S M_{n}\right) \simeq \bigoplus F_{n}^{\mu}
$$

where the sum is over the $\mu$ of depth $\leq n$ such that $\mu_{i}$ is even for all $i$ and $\operatorname{deg}(\mu)=2 k$. It is worth noticing here that the space of polynomials $\oplus_{k} \operatorname{Hom}_{k}\left(S M_{n}\right)$ is isomorphic to the space of polynomial functions on the real symmetric positive definite matrices $S_{n}^{+}$. This set is homogeneous for the action of $\operatorname{GL}(n, \mathbb{R})$, and the stabilizer of the identity matrix is the orthogonal group. So the polynomial functions are the induced module from $O(n)$ to $\mathrm{GL}(n, \mathbb{R})$ of the trivial one. 
The decomposition as a $\operatorname{GL}(n, \mathbb{R}) \times \operatorname{GL}(m, \mathbb{R})$-module of $\operatorname{Hom}_{k}\left(M_{n, m}\right)$ is ([7, Theorem 5.2.7]):

$$
\operatorname{Hom}_{k}\left(M_{n, m}\right) \simeq \bigoplus F_{n}^{\mu} \otimes F_{m}^{\mu}
$$

where the sum is over the $\mu$ of depth at most equal to $\min (m, n)$, and of degree equal to $k$.

From (4) and Frobenius reciprocity, the space of $O(n)$-invariant elements in $F_{n}^{\mu}$ is onedimensional if and only if $\mu_{i}$ is even for all $i$, so, as a $\operatorname{GL}(n, \mathbb{R})$-module:

$$
\operatorname{Hom}_{k}\left(M_{n, m}\right)^{O(m)} \simeq \bigoplus F_{n}^{\mu}
$$

where the sum is over the $\mu$ of depth at most equal to $\min (m, n)$ with all the $\mu_{i} \equiv 0 \bmod 2$ and $\operatorname{deg}(\mu)=k$ (and hence $k$ is even otherwise this space is reduced to 0 ).

Let $p \in \mathcal{G}_{m, n}$ be a $m$-subspace of $\mathbb{R}^{n}$. We associate to $p$ a $n \times m$ matrix $X_{p}$ of an orthonormal basis of $p$. Changing the basis amounts to multiplying $X_{p}$ on the right by an element of $O(m)$, so we can define a mapping:

$$
\begin{aligned}
\pi: \operatorname{Hom}_{2 k}\left(M_{n, m}\right)^{O(m)} & \longrightarrow L^{2}\left(\mathcal{G}_{m, n}\right) \\
f & \longmapsto \pi f: \pi f(p)=f\left(X_{p}\right)
\end{aligned}
$$

which commutes with the action of $O(n)$.

If $X$ is a $n \times m$ matrix, we denote by $X_{1}$ the $m \times m$ matrix $X_{1}=\left(X_{i, j}\right)_{\substack{1 \leq i \leq m \\ 1 \leq j \leq m}}$. The map: $\tau: M_{n, m} \rightarrow S M_{m}$ defined by $\tau(X)=X_{1} X_{1}^{t}$ induces a map:

$$
\tau^{*}: \operatorname{Hom}_{k}\left(S M_{m}\right) \longrightarrow \operatorname{Hom}_{2 k}\left(M_{n, m}\right)^{O(m)}
$$

Note that $\tau^{*}$ sends the degree $k$ polynomials on $S M_{m}$ to degree $2 k$ polynomials on $M_{n, m}$; one checks easily that its image is contained in the $\{1\} \times O(m)$-invariant polynomials.

Frobenius reciprocity theorem [7, 12.1.8] asserts that, since the multiplicity of $F_{m}^{\mu}$ is one in $\operatorname{Hom}_{k}\left(S M_{m}\right)$, there is up to a multiplicative scalar one zonal function in $F_{m}^{\mu}$, which we denote by $C_{\mu}$. We have $C_{\mu}(S)=c_{\mu}\left(\lambda_{1}, \ldots, \lambda_{m}\right)$ where $c_{\mu}$ is a symmetric polynomial homogeneous of degree $k$ (because we can take $S$ diagonal) and $\left(\lambda_{1}, \ldots, \lambda_{m}\right)$ are the eigenvalues of $S$. We normalize $c_{\mu}$ by $c_{\mu}(1,1, \ldots, 1)=1$. The explicit computation of $c_{\mu}$ is given in [8].

Definition 3.4 Let $C_{\mu} \in L^{2}\left(\mathcal{G}_{m, n}\right)$ denote the image of the zonal function $C_{\mu}$ above under the mapping $\pi \tau^{*}$.

The following lemma describes the space of $\operatorname{Stab}\left(p_{0}\right)$-invariant functions in $L^{2}\left(\mathcal{G}_{m, n}\right)$.

Lemma 3.5 The mapping $\pi \tau^{*}$ yields an isomorphism between the $O(m)$-invariant elements of $\operatorname{Hom}_{k}\left(S M_{m}\right)$ and the $\operatorname{Stab}\left(p_{0}\right)$-invariant elements in the image of $\pi$.

Proof: It is easy to see that the $\operatorname{Stab}\left(p_{0}\right)$-invariant polynomial functions in the image of $\pi$ are the symmetric polynomials in the squares $y_{1}, \ldots, y_{m}$ of the cosines of the principal 
angles of $p$ and $p_{0}$. From (2), the $y_{i}$ are the eigenvalues of the symmetric matrix $X_{p, 1} X_{p, 1}^{t} \in$ $S M_{m}$, so such a function is the image by $\pi \tau^{*}$ of an element of $\operatorname{Hom}_{k}\left(\operatorname{SM}_{m}\right)$, which is a polynomial in the eigenvalues of the matrix.

Since the orbits of $O(m)$ on $S_{m}^{+}$are characterized by the set of the eigenvalues of the matrix, the $O(m)$-invariant functions in $\operatorname{Hom}_{k}\left(S M_{m}\right)$ are the symmetric polynomials in the eigenvalues of the matrices (and the mapping $\mathbb{R}\left[Y_{1}, \ldots, Y_{m}\right]^{S_{m}} \rightarrow\left(\oplus_{k} \operatorname{Hom}_{k}\left(S M_{m}\right)\right)^{O(m)}$ is an isomorphism). The possible values for $\left(y_{1}, \ldots, y_{m}\right)$ are $[0,1]^{m}$ so a polynomial which takes the value zero for all $p \in \mathcal{G}_{m, n}$ is zero himself, and the mapping is injective.

We have the following commutative diagram of $O(n)$-modules:

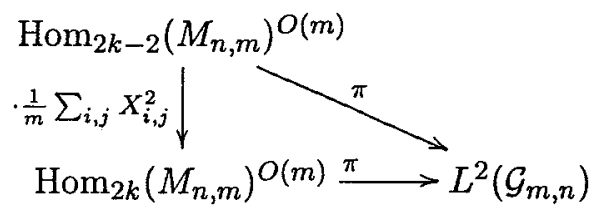

where the vertical arrow is the multiplication by the degree 2 polynomial $\sum_{i, j} X_{i, j}^{2}$. It shows that $\pi\left(\operatorname{Hom}_{2 k-2}\left(M_{n, m}\right)^{O(m)}\right) \subset \pi\left(\operatorname{Hom}_{2 k}\left(M_{n, m}\right)^{O(m)}\right)$. Let us denote

$$
H_{2 k}:=\pi\left(\operatorname{Hom}_{2 k}\left(M_{n, m}\right)^{O(m)}\right) \leq L^{2}\left(\mathcal{G}_{m, n}\right) .
$$

Then

$$
H_{2 k}=H_{2 k-2} \perp W_{2 k}
$$

Proposition 3.6 For a partition $\mu$ of $2 k$, let $\tilde{P}_{\mu}$ be the projection of $C_{\mu} \in H_{2 k}=H_{2 k-2} \oplus$ $W_{2 k}$ to $W_{2 k}$. Then $\tilde{P}_{\mu}$ is a nonzero $\operatorname{Stab}\left(p_{0}\right)$-invariant function in $H_{m, n}^{\mu}$ and hence $\tilde{P}_{\mu}$ is (up to a scalar multiple) the zonal function $P_{\mu}$ above. Moreover, the subspace $H_{m, n}^{\mu}$ is the image by $\pi$ of the (unique) subspace of $\operatorname{Hom}_{2 k}\left(M_{n, m}\right)^{O(m)}$ isomorphic to $V_{n}^{\mu}$.

Proof: It is clear that $\tilde{P}_{\mu}$ is $\operatorname{Stab}\left(p_{0}\right)$-invariant. Since $\tilde{P}_{\mu}$ is $C_{\mu}$ minus a linear combination of $C_{\kappa}$ with $\sum_{i=1}^{n} \kappa_{i}<2 k$, it follows from Lemma 3.5 that $\tilde{P}_{\mu}$ is non zero. Let $X^{\mu}$ be the projection of $F_{n}^{\mu}$ to $W_{2 k}$. Then $X^{\mu}$ is a $O(n)$-submodule of $L^{2}\left(\mathcal{G}_{m, n}\right)$ which contains $\tilde{P}_{\mu}$. By construction and Lemma 3.5 $P_{\mu}$ is the unique $O(m) \times O(n-m)$-invariant function in $X^{\mu}$, which implies that $X^{\mu}$ is an irreducible $O(n)$-module not isomorphic to a submodule of $H_{2 k-2}$. By induction we may assume that $H_{2 k-2} \simeq \oplus V_{n}^{\mu}$ where the sum is over the $\mu$ with $\mu_{i}$ even and $\sum_{i=1}^{m} \mu_{i} \leq 2 k-2$. With Theorem 3.2 one now sees that $X^{\mu}$ is isomorphic to $V_{n}^{\mu}$ and hence $X^{\mu}=\bar{H}_{m, n}^{\mu}$.

It is sometimes more convenient to view the zonal functions as functions on $\mathcal{G}_{m, n} \times \mathcal{G}_{m, n}$. More generally, for $m \leq q \leq \frac{n}{2}$, one can consider the space

$$
\begin{aligned}
& \mathcal{Z}^{m, q}:=\left\{Z: \mathcal{G}_{m, n} \times \mathcal{G}_{q, n} \rightarrow \mathbb{R} \mid Z\left(g(p), g\left(p^{\prime}\right)\right)=Z\left(p, p^{\prime}\right) \forall g \in O(n),\right. \\
& \text { s.t. } \left.Z(p, \cdot) \in L^{2}\left(\mathcal{G}_{q, n}\right), Z\left(\cdot, p^{\prime}\right) \in L^{2}\left(\mathcal{G}_{m, n}\right) \forall p \in \mathcal{G}_{m, n}, p^{\prime} \in \mathcal{G}_{q, n}\right\} .
\end{aligned}
$$


If $m=q$, then any function $Z \in \mathcal{Z}^{m, m}$ yields a zonal function $Z\left(p_{0}, \cdot\right)$ for the stabilizer of $p_{0}$. In particular $\mathcal{Z}^{m, m}$ contains a unique function $P_{\mu}^{m, m}$ with $P_{\mu}(p)=P_{\mu}^{m, m}\left(p_{0}, p\right)$ for all $p \in \mathcal{G}_{m, n}$.

We also need functions $P_{\mu}^{m, q}$ which relate the spaces $L^{2}\left(\mathcal{G}_{m, n}\right)$ and $L^{2}\left(\mathcal{G}_{q, n}\right)$ for $m \leq q \leq$ $\frac{n}{2}$. The fact that the spaces $L^{2}\left(\mathcal{G}_{m, n}\right)$ and $L^{2}\left(\mathcal{G}_{q, n}\right)$ each contain the irreducible module $V_{n}^{\bar{\mu}}$ (for the partitions $\mu$ in even parts and of depth at most $m$ ) with multiplicity one means for $\mathcal{Z}^{m, q}$ that there exists a unique (up to a multiplicative factor, which we choose in the case $m=q$ so that $\left.P_{\mu}^{m, m}(p, p)=1\right)$ non zero element $P_{\mu}^{m, q}$ in $\mathcal{Z}^{m, q}$, such that for all $p \in \mathcal{G}_{m, n}$, $P_{\mu}^{m, q}(p, \cdot)$ belongs to $H_{q, n}^{\mu}$, and for all $p^{\prime} \in \mathcal{G}_{q, n}, P_{\mu}^{m, q}\left(\cdot, p^{\prime}\right)$ belongs to $H_{m, n}^{\mu}$. This element is computed explicitly in [8, Theorem 15.1] and has the general form

$$
P_{\mu}^{m, q}=\sum_{\kappa \leq \mu} \lambda_{\mu, \kappa}^{m, q} C_{\kappa}
$$

where $\kappa \leq \mu$ means that $\kappa_{i} \leq \mu_{i}$ for all $i$. The method used in [8] is to look for eigenvectors of the Laplace-Beltrami operator on the space $\mathbb{R}\left[Y_{1}, \ldots, Y_{m}\right]^{S_{m}}$.

We shall keep the notation $P_{\mu}^{m, m}=P_{\mu}$. Since the $P_{\mu}^{m, q}$ are constant on the orbits of $O(n)$ on $\mathcal{G}_{m, n} \times \mathcal{G}_{q, n}$ and these orbits are characterized by the principal angles between the subspaces, there is a symmetric polynomial $p_{\mu}^{m, q} \in \mathbb{R}\left[Y_{1}, \ldots, Y_{m}\right]^{S_{m}}$ with $P_{\mu}^{m, q}\left(p, p^{\prime}\right)=$ $p_{\mu}^{m, q}\left(y_{1}, \ldots, y_{m}\right)$, where the $y_{i}$ are the squares of the cosines of the principal angles of $p$ and $p^{\prime}$.

In view of our applications, we give the explicit expressions of a few of the polynomials $P_{\mu}^{m, q}\left(p, p^{\prime}\right)=p_{\mu}^{m, q}\left(y_{1}, \ldots, y_{m}\right)$, normalized by $p_{\mu}^{m, m}(1, \ldots, 1)=1$, respectively the polynomials $c_{\mu}$. The indices of $p$ and $c$ are the (at most $m$ ) non zero parts of the corresponding partitions $\mu$. Note that $m \leq q \leq \frac{n}{2}$.

$$
\begin{aligned}
c_{2} & =\frac{1}{m} \sum_{i=1}^{m} y_{i} \\
p_{2}^{m, q} & =\frac{q}{q-n}-\frac{n}{q-n} c_{2} \\
c_{4} & =\frac{3}{m(m+2)}\left(\sum_{1 \leq i \leq m} y_{i}^{2}+\frac{2}{3} \sum_{1 \leq i<j \leq m} y_{i} y_{j}\right) \\
p_{4}^{m, q} & =p_{4}^{\prime} / p_{4}^{\prime}(1 \ldots 1), \quad \text { where } p_{4}^{\prime}=1-\frac{2(n+2)}{q} c_{2}+\frac{(n+2)(n+4)}{q(q+2)} c_{4} \\
c_{22} & =\frac{2}{m(m-1)} \sum_{1 \leq i<j \leq m} y_{i} y_{j} \\
p_{22}^{m, q} & =p_{22}^{\prime} / p_{22}^{\prime}(1 \ldots 1), \quad \text { where } p_{22}^{\prime}=1-\frac{2(n-1)}{q} c_{2}+\frac{(n-1)(n-2)}{q(q-1)} c_{22}
\end{aligned}
$$

It is a classical fact that the $P_{\mu}^{m, q}$ can also be constructed in the following way: Let $\mu$ be a fixed partition, with $\operatorname{depth}(\mu) \leq m$, and let $d$ be the dimension of $V_{n}^{\mu}$. Let $\left(e_{1}, \ldots, e_{d}\right)$ be an orthonormal basis (for the $O(n)$-invariant scalar product $\langle f, g\rangle:=\int_{\mathcal{G}_{m, n}} f(x) g(x) d \mu(x)$, 
where $\mu$ is the Haar measure for $O(n))$ of $H_{m, n}^{\mu}$ and let $\sigma: H_{m, n}^{\mu} \rightarrow H_{q, n}^{\mu}$ be an isomorphism of $O(n)$-modules. Define

$$
Z_{\mu}^{m, q}\left(p, p^{\prime}\right):=\frac{1}{d} \sum_{i=1}^{d} e_{i}(p) \sigma\left(e_{i}\right)\left(p^{\prime}\right) .
$$

This definition is independent of the choice of the basis because another orthonormal basis differs from $\left(e_{1}, \ldots, e_{d}\right)$ by an orthogonal matrix of size $d$; another isomorphism between the subspaces $H_{m, n}^{\mu}$ and $H_{q, n}^{\mu}$ differs from $\sigma$ by a multiplicative scalar. By construction, $Z_{\mu}^{m, q}(p, \cdot) \in H_{q, n}^{\mu}$ and $Z_{\mu}^{m, q}\left(\cdot, p^{\prime}\right) \in H_{m, n}^{\mu}$. If $g \in O(n),\left(g \cdot e_{1}, \ldots, g \cdot e_{d}\right)$ is again an orthonormal basis of $H_{m, n}^{\mu}$, so from the last remark we have $Z_{\mu}^{m, q}\left(g(p), g\left(p^{\prime}\right)\right)=Z_{\mu}^{m, q}\left(p, p^{\prime}\right)$. Moreover, in the case $m=q$, the value $Z_{\mu}(p, p)$ is independent of $p$ because $O(n)$ is transitive on $\mathcal{G}_{m, n}$, and from (14), $Z_{\mu}(p, p)=\left\langle Z_{\mu}(p, p), \mathbf{1}\right\rangle=\frac{1}{d} \sum_{i=1}^{d}\left\langle e_{i}, e_{i}\right\rangle=1$, so $Z_{\mu}=P_{\mu}$.

The expression (14) shows that the operator $f \rightarrow \int Z_{\mu}^{m, q}\left(p, p^{\prime}\right) f\left(p^{\prime}\right) d \mu\left(p^{\prime}\right)$ maps $f \in$ $H_{q, n}^{\mu}$ to $f \in H_{m, n}^{\mu}$, and commutes with the action of $O(n)$.

\section{4. $t$-designs and examples}

In this section, we define the designs in the Grassmannian spaces. Our definition generalizes the notion of spherical designs given in [4].

Definition 4.1 Let $\mathcal{D}$ be a finite subset of $\mathcal{G}_{m, n}$, and let $t$ be an even number. We say that $\mathcal{D}$ is a $t$-design if $\sum_{p \in \mathcal{D}} f(p)=0$ for all $f \in H_{m, n}^{\mu}$ and for all $\mu$ with $2 \leq \operatorname{deg}(\mu) \leq t$.

Proposition 4.2 The following assertions are equivalent, where $t$ is an even integer, and $\mathcal{D} \subset \mathcal{G}_{m, n}$.

1. $\mathcal{D}$ is a $t$-design

2. $\frac{1}{|\mathcal{D}|} \sum_{p \in \mathcal{D}} f(p)=\int_{\mathcal{G}_{m, n}} f(x) d \mu(x)$ for all $f \in H_{t}$, where $H_{t}$ is the subspace of $L^{2}\left(\mathcal{G}_{m, n}\right)$ spanned by all $H_{m, n}^{\mu}$ with $\operatorname{deg}(\mu) \leq t$.

3. For all $\mu$ such that $2 \leq \operatorname{deg}(\mu) \leq t$ and $1 \leq \operatorname{depth}(\mu) \leq m$, there exists $m^{\prime}, \operatorname{depth}(\mu) \leq$ $m^{\prime} \leq m$ such that $\sum_{p \in \mathcal{D}} P_{\mu}^{m^{\prime}, m}\left(p^{\prime}, p\right)=0$ for all $p^{\prime} \in \mathcal{G}_{m^{\prime}, n}$.

4. For all $\mu$ such that $2 \leq \operatorname{deg}(\mu) \leq t$ and $1 \leq \operatorname{depth}(\mu) \leq m$, there exists $m^{\prime}, \operatorname{depth}(\mu) \leq$ $m^{\prime} \leq m$ such that $\sum_{p \in \mathcal{D}} C_{\mu}\left(p^{\prime}, p\right)=\alpha_{\mu}$ for all $p^{\prime} \in \mathcal{G}_{m^{\prime}, n}$, where $\alpha_{\mu}$ is independent of the choice of $p^{\prime} \in \mathcal{G}_{m^{\prime}, n}$.

5. $\sum_{p_{1}, p_{2} \in \mathcal{D}} P_{\mu}\left(p_{1}, p_{2}\right)=0$, for all $\mu$ with $2 \leq \operatorname{deg}(\mu) \leq t$.

Proof: $1 \Rightarrow 2$. Let $f \in H_{t}$. We can write $f=f_{1}+f_{0}$, where $f_{1} \in \oplus_{2 \leq \operatorname{deg}(\mu) \leq t} H_{m, n}^{\mu}$ and $f_{0} \in H_{m, n}^{0 \ldots 0}=\mathbb{R} \mathbf{1}$ (where $\mathbf{1}$ denotes the constant function taking the value one on all the elements of $\left.\mathcal{G}_{m, n}\right)$. So $f=f_{1}+\lambda \mathbf{1}$ and $\int_{\mathcal{G}_{m, n}} f(x) d \mu(x)=\langle f, \mathbf{1}\rangle=\lambda\langle\mathbf{1}, \mathbf{1}\rangle=\lambda$. Since $\sum_{p \in \mathcal{D}} f_{1}(p)=0$, we obtain 2 .

$2 \Rightarrow 3$. We can take $f=P_{\mu}^{m^{\prime}, m}\left(p^{\prime}, \cdot\right)$ and use $P_{\mu}^{m^{\prime}, m}\left(p^{\prime}, \cdot\right) \in H_{m, n}^{\mu}$ which is orthogonal to 1 .

$3 \Rightarrow 4$. Clearly, from (12) we have $C_{\mu}\left(p^{\prime}, p\right)=\sum_{\kappa \leq \mu} \beta_{\kappa, \mu} P_{\kappa}^{m^{\prime}, m}\left(p^{\prime}, p\right)$ for some $\beta_{\kappa, \mu} \in$ $\mathbb{R}$ (also depending on $\left.m^{\prime}, m\right)$. Since $P_{0}^{m^{\prime}, m}\left(p^{\prime}, p\right)=1$, we have $\sum_{p \in \mathcal{D}} C_{\mu}\left(p^{\prime}, p\right)=\beta_{0, \mu}|\mathcal{D}|$. 
$4 \Rightarrow 1$. From (12), the same property holds for $P_{\mu}^{m^{\prime}, m}\left(p^{\prime}, p\right)$; but $P_{\mu}^{m^{\prime}, m}(\cdot, p)$ belongs to $H_{m^{\prime}, n}^{\mu}$, which is orthogonal to $\mathbf{1}$ if $\operatorname{deg}(\mu) \neq 0$. So we have $\sum_{p \in \mathcal{D}} P_{\mu}^{m^{\prime}, m}\left(p^{\prime}, p\right)=0$, for all $p^{\prime} \in \mathcal{G}_{m^{\prime}, n}$ and for all $\mu$ with $2 \leq \operatorname{deg}(\mu) \leq t$. On the other hand, $P_{\mu}^{m^{\prime}, m}\left(p^{\prime}, \cdot\right) \in H_{m, n}^{\mu}$ which is $O(n)$-irreducible, so $H_{m, n}^{\mu}$ is spanned by the images by $O(n)$ of this element. Clearly, $g \cdot P_{\mu}^{m^{\prime}, m}\left(p^{\prime}, \cdot\right)=P_{\mu}^{m^{\prime}, m}\left(g\left(p^{\prime}\right), \cdot\right)$ for all $g \in O(n)$, and we have proved that $\mathcal{D}$ is a $t$-design. $3 \Rightarrow 5$ is obvious, taking $m^{\prime}=m$ (note that we have proved that 3 . is equivalent to 1 ., which does not depend on $m^{\prime}$, so we can freely choose $m^{\prime}=m$ ).

$5 \Rightarrow 3$. We show that 3 . holds for $m^{\prime}=m$, which is enough. Let us define $P:=$ $\sum_{p \in \mathcal{D}} P_{\mu}(p, \cdot)$. In order to prove that $P=0$, we show that $\langle P, P\rangle$ is equal to a constant times $\sum_{p_{1}, p_{2} \in \mathcal{D}} P_{\mu}\left(p_{1}, p_{2}\right)$. It follows from the lemma:

Lemma 4.3 For all $\mu$, there exists a constant $r_{\mu}$ such that, for all $f \in H_{m, n}^{\mu},\left\langle f, r_{\mu} P_{\mu}\right.$ $(p, \cdot)\rangle=f(p)$.

Proof: The operator $f \rightarrow \int P_{\mu}^{m, q}\left(p, p^{\prime}\right) f\left(p^{\prime}\right) d \mu\left(p^{\prime}\right)$ maps $H_{m, n}^{\mu}$ into $H_{q, n}^{\mu}$, and commutes with the action of $O(n)$ (see the end of Section 3). Since $H_{m, n}^{\mu}$ is irreducible, it is homothetic, which is exactly a rephrasement of the lemma.

Corollary 4.4 If $\mathcal{D}$ is a $t$-design, then (3) of Proposition 4.2 holds for all $m^{\prime} \leq m$ and all even partitions $\mu$ of degree $\leq t$ in at most $m^{\prime}$ parts.

Some $t$-designs in $\mathcal{G}_{m, n}$ can arise as orbits under a finite subgroup of $O(n)$ whose representations have special properties.

Theorem 4.5 Let $G$ be a finite subgroup of $O(n)$. Let $m_{0} \leq n / 2$ be a fixed integer. The following properties are equivalent:

1. For all $m \leq m_{0}$, and for all $p \in \mathcal{G}_{m, n}$, the orbit $G \cdot p$ of $p$ under the action of $G$ is a $2 k$-design.

2. The representation of $G$ provided by the subspace of $\operatorname{Hom}_{k}\left(S M_{n}\right)$ isomorphic to $\oplus_{\mu \text { even, depth }(\mu) \leq m_{0}} F_{n}^{\mu}$ contains the trivial character as many times as $O(n)$ itself.

Proof: We consider the mappings:

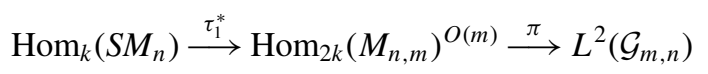

where $\tau_{1}^{*}$ is the $\operatorname{GL}(n, \mathbb{R})$-morphism defined by: $\tau_{1}^{*} P(X)=P\left(X X^{t}\right)$. The surjectivity of $\tau_{1}^{*}$ is a classical result of invariant theory (see [7, Ch. 4]). From the decompositions (4) and (6), we see that its kernel is the sum of the $F_{n}^{\mu}$ with $\operatorname{depth}(\mu)>m$.

Assume the condition 2 . holds. Then, $H_{2 k}^{G}=\mathbb{R} \mathbf{1}$. Let us consider, for $\mu$ with $2 \leq$ $\operatorname{deg}(\mu) \leq 2 k, S:=\sum_{g \in G} g \cdot P_{\mu}(\cdot, p) ; S$ is $G$-invariant and belongs to $H_{m, n}^{\mu}$ so $S=0$. So, for all $p^{\prime} \in \mathcal{G}_{m, n}, \sum_{g \in G} P_{\mu}\left(g\left(p^{\prime}\right), p\right)=0$, which is equivalent to $\sum_{g \in G} P_{\mu}\left(p^{\prime}, g^{-1}(p)\right)=0$, which from Proposition $4.2(3)$. means that $G \cdot p$ is a $2 k$-design.

Conversely, we assume that the subspace of $\operatorname{Hom}_{k}\left(S M_{n}\right)$ isomorphic to $\oplus_{\mu, \operatorname{depth}(\mu) \leq m_{0}} V_{n}^{\mu}$ contains a $G$-invariant element which is not $O(n)$-invariant. We can assume that $k$ is minimal 
and non zero for this property. Let $\mu$ of minimal depth $m\left(m \leq m_{0}\right)$, such that the submodule of $\operatorname{Hom}_{k}\left(S M_{n}\right)$ isomorphic to $F_{n}^{\mu}$ in (4) contains a non zero $G$-invariant element. Because $k$ is minimal, this element belongs to the $O(n)$-submodule of $F_{n}^{\mu}$ isomorphic to $V_{n}^{\mu}$. Hence its image by $\pi \tau_{1}^{*}$ is a non zero $G$-invariant element in $H_{m, n}^{\mu}$ (see Proposition 3.6). Let us call it $f$; there exists $p \in \mathcal{G}_{m, n}$ such that $f(p) \neq 0$, which by definition prevents $G \cdot p$ from being a $2 k$-design.

\section{Remark 4.6}

- When $m_{0}=1$, one recovers the usual criterion for the orbits of a group on the unit sphere modulo $\{ \pm 1\}$ to be spherical designs, since $F_{n}^{(2 k)} \simeq \operatorname{Sym}^{2 k}\left(\mathbb{R}^{n}\right)$.

- The condition 2. in previous theorem is equivalent to the condition: $\operatorname{dim}\left(\left(F_{n}^{\mu}\right)^{G}\right)=1$ for all partition $\mu$, which are even, of degree $2 k$ and depth at most $m_{0}$

- The multiplicity of the trivial character for $O(n)$ in $\oplus F_{n}^{\mu}$ where the sum is over the partitions $\mu$ which are even, of degree $2 k$ and depth at most $m_{0}$ is clearly equal to the number of partitions of $k$ into at most $m_{0}$ parts, $\operatorname{since} \operatorname{dim}\left(F_{n}^{\mu}\right)^{O(n)}=1$ from Frobenius theorem.

We point out two corollaries of Theorem 4.5. The first one is trivial but leads to several interesting examples, and the second one links to a condition on the representations of $G$ which appeared in [9].

Corollary 4.7 Let $G$ be a finite subgroup of $O(n)$. The following properties are equivalent:

1. For all $m \leq n / 2$, and for all $p \in \mathcal{G}_{m, n}$, the orbit $G \cdot p$ of $p$ under the action of $G$ is a $2 k$-design.

2. The representation of $G$ provided by $\operatorname{Hom}_{k}\left(S M_{n}\right)$ contains the trivial character as many times as $O(n)$ itself.

Examples The representation afforded by $\operatorname{Hom}_{k}\left(S M_{n}\right)$ is isomorphic to the representation $\operatorname{Sym}^{k}\left(\operatorname{Sym}^{2}(V)\right)$, where $V$ is the natural representation of the group $G$; standard formulas compute its character from the character of $G$, and hence the multiplicity of the trivial character in it. This computation shows that the condition 2. of Theorem 4.5 holds for $\operatorname{Aut}\left(D_{4}\right)$ and $k \leq 2, \operatorname{Aut}\left(E_{6}\right)$ and $k \leq 2, \operatorname{Aut}\left(E_{7}\right)$ and $k \leq 2, \operatorname{Aut}\left(E_{8}\right)$ and $k \leq 3, \operatorname{Aut}\left(K_{12}\right)$ and $k=1, \operatorname{Aut}\left(B W_{16}\right)$ and $k \leq 3, \operatorname{Aut}\left(\Lambda_{24}\right)$ the Leech lattice and $k \leq 5$. In the cases $D_{4}, E_{6}, E_{7}, E_{8}, B W_{16}$ and $\Lambda_{24}$, the lowest degree $G$-invariant polynomial appears for a partition $\mu$ of depth equal to 1 , so the degree of the design afforded by the $m$-sections of the lattice is the same as the degree of the design on the minimal vectors (which is known from $[1,11])$. In the case of the Coxeter-Todd lattice $K_{12}$, the minimal vectors form a 4-design, but a $G$-invariant polynomial appears for $\mu=(2,2,0, \ldots, 0)$. One can check that the two orbits of $G$ on the minimal planes of the lattice (of cardinality 5040 and 126) do not form 4-designs (nor their union).

Corollary 4.8 Let $G$ be a finite subgroup of $O(n)$. The following properties are equivalent: 1. For all $p \in \mathcal{G}_{1, n} \cup \mathcal{G}_{2, n}$, the orbit $G \cdot p$ of $p$ under the action of $G$ is a $2 k$-design. 
2. The representation of $G$ provided by $\operatorname{Sym}^{2}\left(\operatorname{Sym}^{k}\left(\mathbb{R}^{n}\right)\right)$ contains the trivial character as many times as $O(n)$ itself.

Proof: There is an isomorphism of $\operatorname{GL}(n, \mathbb{R})$-modules between the spaces $\mathrm{Hom}_{2 k}$ $\left(M_{n, 2}\right)^{O(2)}$ and $\operatorname{Sym}^{2}\left(\operatorname{Sym}^{k}\left(\mathbb{R}^{n}\right)\right)\left(\right.$ if $X \in M_{n, 2}$, consider $S_{j}=X_{j, 1}+i X_{j, 2}$ and $T_{j}=X_{j, 1}$ $i X_{j, 2}$; one easily sees that the $O(2)$-invariant homogeneous of degree $2 k$ polynomials in the $X_{i, j}$ are the polynomials which are of degree $k$ in $S_{i}$ and in $T_{j}$ and invariant under the exchange $\left.\left(S_{i}, T_{i}\right)\right)$.

The condition 2 of Corollary 4.8 is the same as the condition (ii) of [9, Theorem 2.6]. In particular, the almost irreducible groups listed in [9] give rise to 4-designs in both the grassmannians $\mathcal{G}_{1, n}$ and $\mathcal{G}_{2, n}$. When $G$ is the automorphism group of a lattice $L$, the lattice is strongly 1- and 2-perfect (see next section).

\section{The Rankin function $\gamma_{n, m}$}

Beside the classical Hermite function $\gamma$ ( $=\gamma_{1}$ in what follows), Rankin [10] defined a collection of functions $\gamma_{m}$, in the following way: let $L$ be a lattice in a Euclidean space $E$, endowed with a scalar product denoted $x \cdot y$, and $m$ an integer in $\{1, \ldots, n\}$. One defines

$$
\delta_{m}(L)=\inf _{p \in L(m)} \operatorname{det} p
$$

in which $L(m)$ stands for the set of $m$-dimensional sublattices of $L$, and

$$
\gamma_{m}(L)=\delta_{m}(L) /(\operatorname{det} L)^{\frac{m}{n}}
$$

Thus, for $m=1, \gamma_{1}(L)$ is the classical Hermite invariant of $L$. It can be proved ([10]) that $\gamma_{m}$ is bounded as a function on the set of $n$-dimensional positive definite lattices. The supremum, which actually is a maximum, is denoted by $\gamma_{m, n}$. In [3], a notion of $m$-perfection and $m$-eutaxy were proposed, which we recall below, and a characterization of the local maxima of $\gamma_{m}$ was derived.

We define the set of minimal $m$-sections of $L$ as

$$
\mathcal{S}_{m}(L)=\left\{p \in L(m) \mid \operatorname{det} p=\delta_{m}(L)\right\}
$$

which is a finite set. If $p$ is a $m$-section, we denote by $\mathrm{pr}_{p}$ the orthogonal projection on $p$, seen as an element of the space $\operatorname{End}^{s}(E)$ of the symmetric endomorphisms of the Euclidean space $E$. We recall the definitions given in [3]:

\section{Definition 5.1}

1. A lattice $L$ is called $m$-perfect if the endomorphisms $\operatorname{pr}_{p}$ generate $\operatorname{End}^{s}(E)$.

2. A lattice $L$ is $m$-eutactic if there exist positive coefficients $\lambda_{p}, p \in S_{m}(L)$ such that $\sum_{p \in \mathcal{S}_{m}(L)} \lambda_{p} \mathrm{pr}_{p}=I d$.

3. A lattice $L$ is called $m$-extreme, if $\gamma_{m}$ achieves a local maximum at $L$. 
Recall ([3, Theorem 3.2.3]) that $L$ is $m$-extreme if and only if $L$ is both $m$-perfect and $m$-eutactic.

\section{Strongly $m$-perfect lattices}

In this section, we study the connection between the notion of design on grassmannians introduced in Section 4, and the Rankin invariant of lattices. Our goal is to generalise the result of Boris Venkov, which asserts that, if the minimal vectors of a lattice are a 4-spherical design, i.e. a 4-design in $\mathcal{G}_{1, n}$, then this lattice is a local maximum of the Hermite function ([12, Theorem 6.4]).

Definition 6.1 Let $L$ be a lattice of dimension $n$, and let $S_{m}(L) \subset \mathcal{G}_{m, n}$ be the set of its minimal sections of dimension $m$. We say that $L$ is strongly $m$-perfect if $S_{m}(L)$ is a 4-design in the sense of Definition 4.1.

Examples The lattices $D_{4}, E_{6}, E_{7}, E_{8}, B W_{16}, \Lambda_{24}$ are strongly $m$-perfect for all $m \leq n / 2$ from Section 4. The Coxeter-Todd lattice, which is strongly 1-perfect [1], is not strongly 2perfect (see the examples in Section 4). Despite of that, the associated orthogonal projections span the space of symmetric endomorphisms, and the lattice is 2-extreme.

Theorem 6.2 If $L$ is strongly m-perfect, then it is m-extreme, i.e. it achieves a local maximum of the Rankin function $\gamma_{m}$.

Proof: We use the characterization of $m$-extreme lattices recalled in Section 5. If $p$ belongs to $\mathcal{G}_{m, n}$, we denote, as in Section 5, $\mathrm{pr}_{p}$ the orthogonal projection on $p$. The space $\operatorname{End}^{S}(E)$ is endowed with the usual scalar product $A \cdot B=\operatorname{trace}(A B)$. The main ingredient for the proof is the following remark: for all $p, p^{\prime}$ in $\mathcal{G}_{m, n}, m C_{2}\left(p, p^{\prime}\right)=\operatorname{pr}_{p} \cdot \operatorname{pr}_{p}^{\prime}$, and it is obvious in view of Section 2 and from the expression of $c_{2}$ (13).

We first prove that, if $S_{m}(L)$ is a 2-design, then $L$ is $m$-eutactic. To that end, we prove that $\sum_{p \in S_{m}(L)} \operatorname{pr}_{p}=\lambda I d$ for some $\lambda \in \mathbb{R}$. It is worth noticing that $\lambda$ is forced to be positive because $\operatorname{pr}_{p} \cdot I d=m$, and hence $\lambda=\left|S_{m}(L)\right| m / n$. Because End ${ }^{S}(E)$ is generated by all the $p r_{p^{\prime}}$ when $p^{\prime}$ runs over $\mathcal{G}_{m, n}$, it is enough to prove that, for all $p^{\prime} \in \mathcal{G}_{m, n}, \sum_{p \in S_{m}(L)} \mathrm{pr}_{p} \cdot p r_{p^{\prime}}=$ $\lambda I d \cdot p r_{p^{\prime}}$, which is equivalent to $\sum_{p \in S_{m}(L)} C_{2}\left(p, p^{\prime}\right)=\lambda$. But this last condition is exactly the condition 4. of Proposition 4.2.

Let $C:=\left(C_{2}\left(p, p^{\prime}\right)\right)_{p, p^{\prime} \in S_{m}(L)}$. Since the Gram matrix of the projections $\operatorname{pr}_{p}$ equals $m C$, the $m$-perfection is equivalent to the property that $C$ has rank equal to $\operatorname{dim} \operatorname{End}^{s}(E)=$ $n(n+1) / 2$. Let $J$ denote the matrix with all coefficients equal to 1 . The property that $S_{m}(L)$ is a 4-design actually leads to a linear relation between $C, C^{2}$ and $J$.

Lemma 6.3 Assume $S_{m}(L)$ is a 4-design and let $C:=\left(C_{2}\left(p, p^{\prime}\right)\right)_{p, p^{\prime} \in S_{m}(L)}$. Let $d:=$ $\operatorname{dim}\left(H_{m, n}^{2}\right)=n(n+1) / 2-1$ and let $s_{m}:=\left|S_{m}(L)\right|$. Then

$$
C^{2}=s_{m}\left(\frac{n-m}{n d} C+\frac{m^{2} d+m^{2}-n m}{n^{2} d} J\right) .
$$


Proof: We introduce the matrix $P:=\left(P_{2}\left(p, p^{\prime}\right)\right)_{p, p^{\prime} \in S_{m}(L)}$ where $P_{2}=Z_{2}^{m, m}$ is defined in (14) and we compute $P^{2}$. For all $p, p^{\prime}$ in $S_{m}(L)$,

$$
\begin{aligned}
P^{2}\left(p, p^{\prime}\right) & =\sum_{p^{\prime \prime} \in S_{m}(L)} P_{2}\left(p, p^{\prime \prime}\right) P_{2}\left(p^{\prime \prime}, p^{\prime}\right) \\
& =\frac{1}{d^{2}} \sum_{p^{\prime \prime} \in S_{m}(L)} \sum_{i, j} e_{i}(p) e_{i}\left(p^{\prime \prime}\right) e_{j}\left(p^{\prime \prime}\right) e_{j}\left(p^{\prime}\right) \\
& =\frac{1}{d^{2}} \sum_{i, j} e_{i}(p) e_{j}\left(p^{\prime}\right) \sum_{p^{\prime \prime} \in S_{m}(L)} e_{i}\left(p^{\prime \prime}\right) e_{j}\left(p^{\prime \prime}\right) .
\end{aligned}
$$

Clearly, the function $p \rightarrow e_{i}(p) e_{j}(p)$ belongs to $H_{4}$. According to the decomposition $H_{4}=\mathbb{R} \mathbf{1} \perp H_{m, n}^{2} \perp H_{m, n}^{22} \perp H_{m, n}^{4}, e_{i} e_{j}=\lambda_{0} \mathbf{1}+f_{2}+f_{22}+f_{4}$. Because $S_{m}(L)$ is assumed to be a 4-design, the sums $\sum_{p^{\prime \prime} \in S_{m}(L)} f_{2}\left(p^{\prime \prime}\right), \sum_{p^{\prime \prime} \in S_{m}(L)} f_{22}\left(p^{\prime \prime}\right), \sum_{p^{\prime \prime} \in S_{m}(L)} f_{4}\left(p^{\prime \prime}\right)$ are equal to zero. Moreover, since $\delta_{i, j}=\left\langle e_{i}, e_{j}\right\rangle=\left\langle e_{i} e_{j}, \mathbf{1}\right\rangle=\lambda_{0}\langle\mathbf{1}, \mathbf{1}\rangle=\lambda_{0}$,

$$
\begin{aligned}
P^{2}\left(p, p^{\prime}\right) & =\frac{1}{d^{2}} \sum_{i} e_{i}(p) e_{i}\left(p^{\prime}\right) s_{m} \\
& =\frac{s_{m}}{d} P\left(p, p^{\prime}\right)
\end{aligned}
$$

and we have proved the matrix relation $P^{2}=\frac{s_{m}}{d} P$. In order to prove the lemma, we need to compute $C$ in terms of $P$ and $J$. From (13) we have

$$
C=\frac{m}{n} J+\frac{n-m}{n} P .
$$

Finally, the property that $J P=P J=0$, which holds because $S_{m}(L)$ is a 2-design, together with (21) and (22) ends the proof of the lemma.

The matrices $C, C^{2}, J$ are real symmetric matrices which pairwise commute, so they are simultaneously diagonalizable. The eigenvalues of $J$ are $s_{m}$ with multiplicity one, and 0 . Let us denote $\lambda_{1}, \lambda_{2}, \ldots$ the eigenvalues of $C$, where $\lambda_{1}$ corresponds to the eigenvector $(1,1, \ldots, 1)$. From the identity $C J=m / n J^{2}=s_{m} m / n J$, we derive $\lambda_{1}=s_{m} m / n$. From the identity (19), for $i \geq 2, \lambda_{i}^{2}=s_{m} \frac{n-m}{n d} \lambda_{i}$, so $\lambda_{i}=0$ or $\lambda_{i}=s_{m} \frac{n-m}{n d}$. Let us denote $k$ the multiplicity of $s_{m} \frac{n-m}{n d}$. We have trace $(C)=s_{m}=s_{m} \frac{m}{n}+k s_{m} \frac{n-m}{n d}$, from which $k=d$. Finally, the rank of the matrix $C$ is equal to $1+d=n(n+1) / 2=\operatorname{dim}\left(\operatorname{End}^{s}(E)\right)$, which ends the proof of the theorem.

\section{Bounds for the minima}

In this section we obtain analogous bounds as the ones given in [12 Théorème 10.4]. There it is shown that for a strongly 1-perfect lattice $L$, the product of the minima of the lattice $L$ and its dual lattice satisfies $\min (L) \min \left(L^{*}\right) \geq \frac{n+2}{3}$.

Using analogous methods we obtain 
Theorem 7.1 Let L be a strongly 2-perfect lattice of dimension $n$. Let $m$ be its minimum, let $d$ be the minimal determinant of a 2-dimensional sublattice of $L$ and $m^{\prime}$ be the minimum of the dual lattice $L^{*}$. Then

$$
d m^{\prime} \geq m \frac{n+2}{4}
$$

Proof: Let $\alpha$ be a minimal vector of $L^{*}$ and $\mathcal{D}$ denote the set of minimal 2-sections of $L$. We consider $p^{\prime}:=\langle\alpha\rangle \in \mathcal{G}_{1, n}$ and $\mathcal{D} \subset \mathcal{G}_{2, n}$. The polynomials $p_{2}^{1,2}$ and $p_{4}^{1,2}$ are polynomials in one variable $y_{1}$ which can be calculated with Eq. (2): Let $p:=\left\langle e_{1}, e_{2}\right\rangle \in \mathcal{D}$ be a minimal 2 -section of determinant $d$ in $L$. Then the principal angle between $p$ and $p^{\prime}$ is

$$
\begin{aligned}
& (\alpha, \alpha)^{-1} d^{-1}\left(\left(\alpha, e_{1}\right), \quad\left(\alpha, e_{2}\right)\right)\left(\begin{array}{cc}
\left(e_{2}, e_{2}\right) & -\left(e_{1}, e_{2}\right) \\
-\left(e_{1}, e_{2}\right) & \left(e_{1}, e_{1}\right)
\end{array}\right)\left(\begin{array}{c}
\left(\alpha, e_{1}\right) \\
\left(\alpha, e_{2}\right)
\end{array}\right) \\
& =(\alpha, \alpha)^{-1} d^{-1}\left\|\left(\alpha, e_{1}\right) e_{2}-\left(\alpha, e_{2}\right) e_{1}\right\|^{2} .
\end{aligned}
$$

To shorten the notation we define $N(p):=\left\|\left(\alpha, e_{1}\right) e_{2}-\left(\alpha, e_{2}\right) e_{1}\right\|^{2}$. Then $N(p)$ is the squared length of a vector in $L$ and hence it is either 0 or $\geq \min (L)=m$. Using Corollary 4.4 we calculate with the Eqs. (13)

$$
\sum_{p \in \mathcal{D}} N(p)=\frac{2}{n}(\alpha, \alpha) d|\mathcal{D}|
$$

and

$$
\sum_{p \in \mathcal{D}} N(p)^{2}=\frac{8}{n(n+2)}(\alpha, \alpha)^{2} d^{2}|\mathcal{D}|
$$

From this we find that

$$
\begin{aligned}
\sum_{p \in \mathcal{D}} N(p)^{2}-m N(p) & =\frac{8}{n(n+2)}(\alpha, \alpha)^{2} d^{2}|\mathcal{D}|-m \frac{2}{n}(\alpha, \alpha) d|\mathcal{D}| \\
& =\frac{2 m}{n}(\alpha, \alpha) d|\mathcal{D}|\left(\frac{4}{m(n+2)}(\alpha, \alpha) d-1\right)
\end{aligned}
$$

Since the left hand side is a sum over nonnegative real numbers, also the right hand side is a nonnegative number. Hence $\frac{4}{m(n+2)}(\alpha, \alpha) d \geq 1$ or equivalently $(\alpha, \alpha) d \geq \frac{m(n+2)}{4}$.

Examples For $L=D_{4}$ one has $m=2$ hence $d m^{\prime} \geq \frac{2(4+2)}{4}=3$, which is sharp since $d=3$ and $m^{\prime}=1$. For $L=E_{8}$ the formula yields the bound 5 , whereas $d m^{\prime}=6$, for $K_{12}$ one finds $16 \geq 14$ and for $B W_{16}$ one has $24 \geq 18$. Theorem 7.1 also allows to conclude that certain lattices are not strongly 2-perfect. For instance for $L=A_{4}$ one finds $d m^{\prime}=3 \frac{4}{5}$ which is not $\geq \frac{2(4+2)}{4}=3$. 
In the case $L$ is integral, one can take a minimal vector $\alpha \in L$ instead of $\alpha \in L^{*}$ in the proof of Theorem 7.1, and get the following lower bound:

Corollary 7.2 Let $L$ be an integral strongly 2-perfect lattice of dimension $n$ and put $d:=\delta_{2}(L)$. Then

$$
d \geq \frac{n+2}{4}
$$

Theorem 7.3 Let $L$ be an integral strongly 2-perfect lattice of dimension $n$ and put $d:=\delta_{2}(L)$ and $d^{\prime}:=\delta_{2}\left(L^{*}\right)$. Then

$$
d d^{\prime} \geq \frac{(n+2)(n-1)}{2(3 n-2) \delta}
$$

where $\delta \in \mathbb{N}$ is the least common multiple of the denominator of the Gram matrices of the minimal 2-sections on $L^{*}$.

Proof: We denote by $\mathcal{D}$ the set of minimal 2-sections of $L$. Let $p^{\prime}:=\left\langle f_{1}, f_{2}\right\rangle \subset L^{*}$ be a minimal plane of $L^{*}$ of determinant $d^{\prime}$ and let $p:=\left\langle e_{1}, e_{2}\right\rangle \in \mathcal{D}$.

Denote by $y_{1}, y_{2}$ the squares of the cosines of the principal angles between $p$ and $p^{\prime}$. Then

$$
y_{1} y_{2}=\frac{1}{d d^{\prime}} \operatorname{det}\left(\left(\begin{array}{ll}
\left(e_{1}, f_{1}\right) & \left(e_{1}, f_{2}\right) \\
\left(e_{2}, f_{1}\right) & \left(e_{2}, f_{2}\right)
\end{array}\right)\right)^{2}=\frac{1}{d d^{\prime}} D\left(p, p^{\prime}\right)
$$

with $D\left(p, p^{\prime}\right) \in \mathbb{Z}$ and

$$
y_{1}+y_{2}=\frac{1}{d d^{\prime}} N\left(p, p^{\prime}\right)
$$

where $N\left(p, p^{\prime}\right)$ can be calculated from (2) and is in $\frac{1}{\delta} \mathbb{Z}$ where $\delta$ is the denominator of the Gram matrix of $p^{\prime}$.

From Eqs. (13) we find that

$$
\begin{aligned}
\sum_{p \in \mathcal{D}} N\left(p, p^{\prime}\right) & =d d^{\prime}|\mathcal{D}| \frac{4}{n} \\
\sum_{p \in \mathcal{D}} D\left(p, p^{\prime}\right) & =d d^{\prime}|\mathcal{D}| \frac{2}{n(n-1)} \\
\sum_{p \in \mathcal{D}}\left(N\left(p, p^{\prime}\right)\right)^{2} & =\left(d d^{\prime}\right)^{2}|\mathcal{D}| \frac{8(3 n-2)}{n(n+2)(n-1)}
\end{aligned}
$$


From this one gets that

$$
\sum_{p \in \mathcal{D}}\left(N\left(p, p^{\prime}\right)\right)^{2}-\frac{1}{\delta} N\left(p, p^{\prime}\right)=d d^{\prime}|\mathcal{D}| \frac{4}{\delta n}\left(d d^{\prime} \frac{2(3 n-2) \delta}{(n+2)(n-1)}-1\right) \geq 0
$$

and hence $d d^{\prime} \geq \frac{(n+2)(n-1)}{2(3 n-2) \delta}$.

\section{Acknowledgments}

The exchange leading to this work has been enabled by a PROCOPE project. We thank the European community for this financial support.

\section{References}

1. C. Bachoc and B. Venkov, "Modular forms, lattices and spherical designs," in "Réseaux euclidiens, "designs" sphériques et groupes," in L'Enseignement Mathématique J. Martinet (Éd.), Monographie no. 37, Genève (2001), to appear.

2. J.H. Conway, R.H. Hardin, and N.J.A. Sloane, "Packing lines, planes, etc., packings in Grassmannian spaces," Experimental Mathematics 5 (1996), 139-159.

3. R. Coulangeon,"Réseaux k-extrêmes," Proc. London Math. Soc. 73 (3) (1996), 555-574.

4. P. Delsarte, J.M. Goethals, and J.J. Seidel, “Spherical codes and designs,” Geom. Dedicata 6 (1977), 363-388.

5. W. Fulton and J. Harris, Representation Theory, a First Course, GTM, Vol. 129, Springer, 1991.

6. G.H. Golub and C.F. Van Loan, Matrix Computations, 2nd edn., John Hopkins University Press, 1989.

7. R. Goodman and N.R. Wallach, Representations and Invariants of the Classical Groups, Encyclopedia of Mathematics and its Applications, Vol. 68, Cambridge University Press, 1998.

8. A.T. James and A.G. Constantine, "Generalized Jacobi polynomials as spherical functions of the Grassmann manifold,” Proc. London Math. Soc. 29(3) (1974), 174-192.

9. W. Lempken, B. Schröder, and P.H. Tiep, "Symmetric squares, spherical designs and lattice minima," J. Algebra 240 (2001), 185-208.

10. R.A. Rankin, "On positive definite quadratic forms," J. London Math. Soc. 28 (1953), 309-314.

11. B. Venkov, "Even unimodular extremal lattices," Proc. Steklov Inst. Math. 165 (1984), 47-52.

12. B. Venkov, "Réseaux et designs sphériques," in "Réseaux euclidiens, "designs" sphériques et groupes," J. Martinet (Éd.), L'Enseignement Mathématique, Monographie no 37, Genève (2001), to appear. 